\title{
Lexical-perceptual integration influences sensorimotor adaptation in speech
}

\author{
Nicolas J. Bourguignon ${ }^{1,2,3}$, Shari R. Baum ${ }^{3,4}$ and Douglas M. Shiller ${ }^{1,2,3 *}$ \\ ${ }^{1}$ Research Center, Sainte-Justine Hospital, Université de Montréal, Montreal, OC, Canada \\ 2 School of Speech Pathology and Audiology, Université de Montréal, Montreal, QC, Canada \\ ${ }^{3}$ Centre for Research on Brain, Language and Music, McGill University, Montreal, OC, Canada \\ ${ }^{4}$ School of Communication Sciences and Disorders, McGill University, Montreal, OC, Canada
}

\section{Edited by:}

Dave Saint-Amour, Université du Québec à Montréal, Canada

Reviewed by:

Shanqing Cai, Boston University, USA

Caroline A Niziolek, University of California, San Francisco, USA

\section{${ }^{*}$ Correspondence:}

Douglas M. Shiller, Research Center, Sainte-Justine Hospital, Université de Montréal, 7077 Parc avenue, room 3001-31, Montreal, QC H3N $1 X 7$, Canada

e-mail:douglas.shiller@umontreal.ca

A combination of lexical bias and altered auditory feedback was used to investigate the influence of higher-order linguistic knowledge on the perceptual aspects of speech motor control. Subjects produced monosyllabic real words or pseudo-words containing the vowel $[\varepsilon]$ (as in "head") under conditions of altered auditory feedback involving a decrease in vowel first formant (F1) frequency. This manipulation had the effect of making the vowel sound more similar to [I] (as in "hid"), affecting the lexical status of produced words in two Lexical-Change (LC) groups (either changing them from real words to pseudo-words: e.g., less-liss, or pseudo-words to real words: e.g., kess—kiss). Two Non-Lexical-Change (NLC) control groups underwent the same auditory feedback manipulation during the production of $[\varepsilon]$ real- or pseudo-words, only without any resulting change in lexical status (real words to real words: e.g., mess-miss, or pseudo-words to pseudo-words: e.g., ness-niss). The results from the LC groups indicate that auditory-feedback-based speech motor learning is sensitive to the lexical status of the stimuli being produced, in that speakers tend to keep their acoustic speech outcomes within the auditory-perceptual space corresponding to the task-related side of the word/non-word boundary (real words or pseudo-words). For the NLC groups, however, no such effect of lexical status is observed.

Keywords: speech production, sensorimotor integration, lexical effect, altered auditory feedback, language processing

\section{INTRODUCTION}

Linguistic processing of the speech acoustic signal is a notoriously challenging phenomenon: speakers and listeners must selectively navigate ambiguous auditory scenes in real time and filter out irrelevant noise from the ambient environment (Zion Golumbic et al., 2012). To achieve this, the nervous system must be able to extract and integrate various types of perceptual, motor and linguistic information from the incoming speech stream. Instances of information integration across the perceptual and motor domains have been particularly well documented in both speech perception and production (see below), but systematic attempts to understand how these various components interact with each other as well as with more abstract levels of linguistic representation have just begun to take shape (Hickok et al., 2011; Hickok, 2012). The present study aims to contribute to this effort by exploring the possible top-down influence of linguistic information - in this case, the lexical status of words-on the sensorimotor networks supporting spoken language production.

In language perception, it has long been demonstrated that linguistic context can determine how speech sounds or words are interpreted (Miller et al., 1951; Warren and Sherman, 1974; Ganong, 1980). Amongst the most illustrative examples of this is Ganong's (1980) lexical effect on phoneme identification, whereby subjects are presented with a phonetically ambiguous consonant (e.g., between a $[\mathrm{d}]$ and $\mathrm{a}[\mathrm{t}]$ as determined by voice onset time), occurring in a lexical context (e.g., "-ash") such that one interpretation is consistent with a real-word (e.g., "dash") and the other maps onto a pseudo-word ("tash"). Ganong's (1980) study and later replications indicate that the identification of a target sound is biased toward real words (Figure 1), effectively shifting phonemic boundaries in favor of existing lexical entries (Connine and Clifton, 1987; Burton et al., 1989; Pitt, 1995).

These findings have been taken to suggest a direct influence of lexical knowledge on the classification of phonetic categories, however the way in which phonetic-lexical integration takes place and at which stage of the recognition process remains a matter of debate (Fox, 1984; Connine and Clifton, 1987; Pitt and Samuel, 1993; Pitt, 1995; Myers and Blumstein, 2008). One key question is whether this lexical effect emerges from a direct influence of lexical entries on the perception of phonetic input (Figure 2A), or whether lexical knowledge is used at a later stage for the purpose of explicit perceptual decision-making (Figure 2B). Methodologically, disentangling this issue has been complicated by the fact that the lexical effect has been investigated through observation of participants' explicit classification of auditory stimuli (i.e., phoneme identification tasks). Such overt classificatory tasks are not typical of speech perception under naturally occurring conditions, hence it remains unclear to what extent lexical knowledge plays a role in everyday phonetic processing (Fox, 1984). If the lexical effect is primarily related to 
overt decision-making ability, and not to the perception of speech acoustic properties per se, then doing away with any such explicit classificatory task would be expected to reduce or eliminate the influence of lexical knowledge on speech perception. Recent appeal to neuroimaging methods such as fMRI, MEG, and/or EEG (Gow et al., 2008; Myers and Blumstein, 2008) has helped to address this question. In particular, Gow et al. (2008) were able

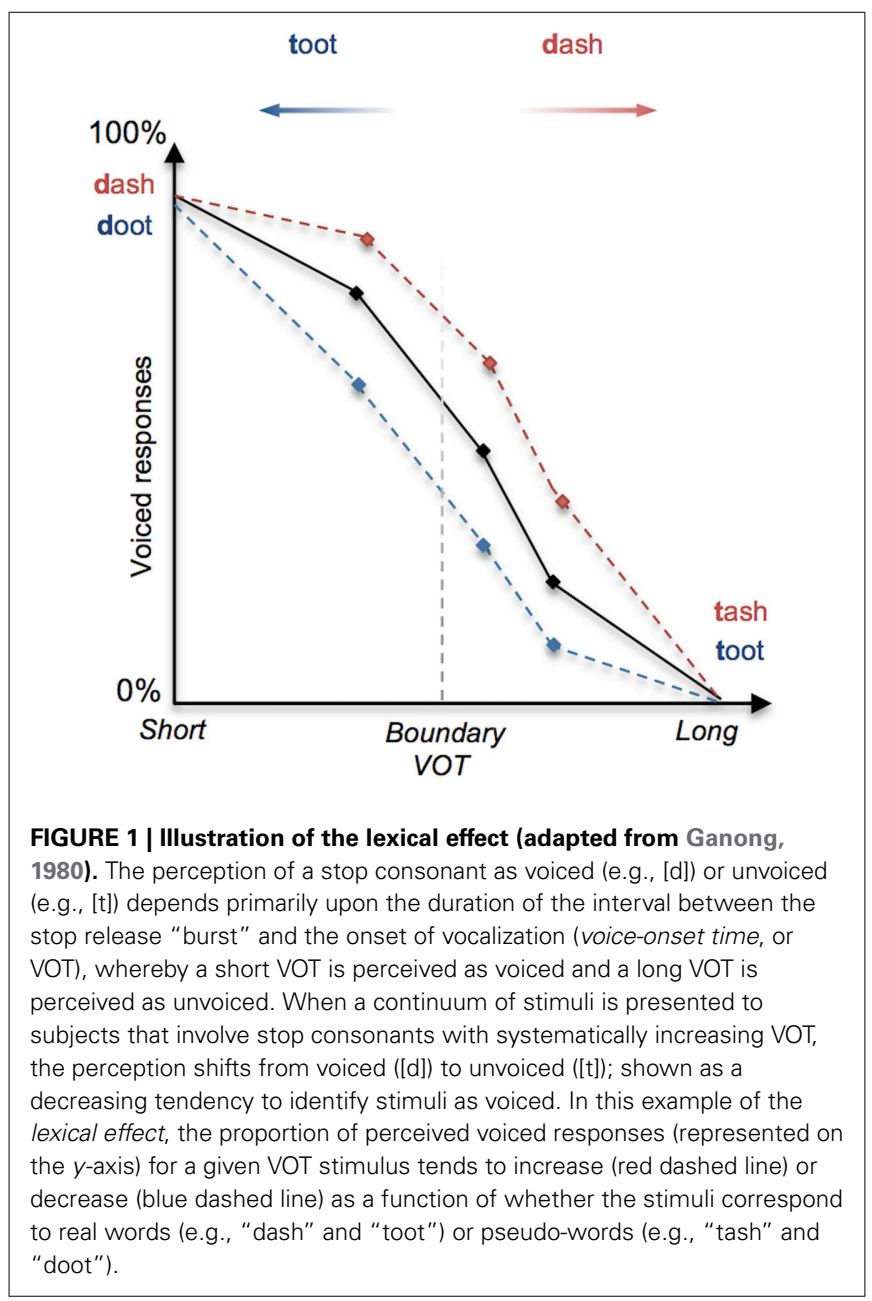

to gain spatially and temporally fine-grained neurophysiological evidence supporting a causal influence of brain areas involved in lexical knowledge on those supporting first-order phonetic processing of ambiguous speech stimuli (similar to those used by Ganong, 1980) before areas shown to be involved in perceptual decision-making were recruited (e.g., the left inferior frontal gyrus, see Burton et al., 2000). These findings thus support the notion of a direct top-down influence of lexical representations on speech perception.

Efficient speech production is also known to result from the integration of articulatory motor control with both perceptual and linguistic information, but the influence of these cues upon speech motor processes has so far been investigated using largely non-overlapping approaches (Hickok, 2012 see also Hickok et al., 2011). On the one hand, numerous studies have emphasized that acoustic-phonetic goals guide articulatory control (Houde and Jordan, 1998; Tourville et al., 2008; Shiller et al., 2009). A standard way to demonstrate this influence is to perturb participants' auditory feedback as they produce syllables or words (e.g., "head") in such a way that a change in acoustic properties (e.g., a decrease in the vowel first formant frequency, or F1) results in the perception of a different speech sound (e.g., a vowel closer to [I], as in "hid"). The perceived deviation of the produced speech acoustic signal from the acoustic target provokes a compensatory change in participants' speech output (e.g., an F1 increase), indicating a reliance on the phonetic processing of auditory feedback in guiding and adapting future productions (Houde and Jordan, 1998; Shiller et al., 2009).

On the other hand, psycholinguistic research has pointed to the influence of abstract lexical information on speakers' articulatory patterns (Levelt, 1983, 1989), in particular their tendency to substitute phonemes more often in words or word strings when these substitutions yield real words (barn door-darn bore) as opposed to pseudo-words (dart board_* bart doard, Baars et al., 1975; Costa et al., 2006; Oppenheim and Dell, 2008). Interestingly, this so-called lexical bias echoes Ganong's lexical effect in showing that the speech production system is biased toward real words relative to pseudo-words.

It is evident from these separate demonstrations that lexical status influences the perception of speech sounds, and that both auditory-perceptual and lexical information play a role in the

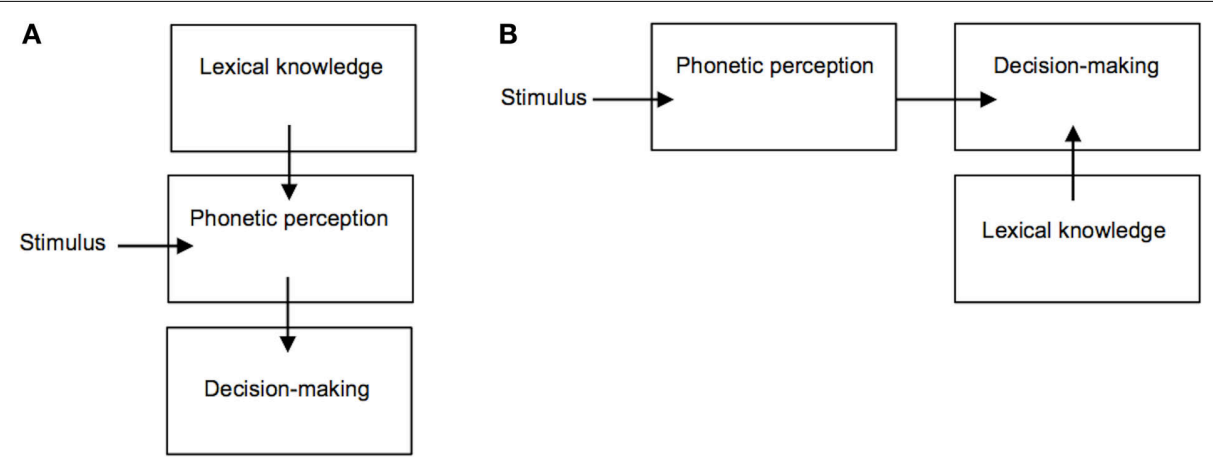

FIGURE 2 | Competing accounts of the lexical effect on phoneme perception. (A) Lexical knowledge directly influences phonetic perception; (B) Lexical knowledge is used at a later stage for the purpose of explicit decision-making. 
neural control of speech production. What remains unknown is the extent to which lexical and phonetic information may interact during the control of speech production. We investigated this question by combining a variant of Ganong's (1980) lexical manipulation with a paradigm of sensorimotor adaptation to altered auditory feedback during speech production. Specifically, we compared participants' motor adaptation to a perceived decrease in F1 frequency during production of the vowel [E] (resulting in a vowel perceived to be closer to [I]) under two different conditions. In the Lexical-Change (LC) condition, participants produced a series of $[\varepsilon]$ real-words (e.g., less) or pseudo-words (e.g., kess) that were chosen in such a way that the feedback alteration resulted in the perception of stimuli as having a different lexical status (i.e., a real-word perceived as a pseudo-word, or a pseudo-word perceived as a real-word). On the basis of previous demonstrations of the lexical effect (Baars et al., 1975; Ganong, 1980), we hypothesized that in participants' phonetic perception of their own speech auditory feedback, the phonetic boundary would be biased in accordance with lexical status (with the boundary shifting in the direction of the vowel in the non-lexical item, thus enlarging the area along the continuum containing the real-word) and that this bias would be reflected in their patterns of articulatory compensation (see Figure 3). An interaction between phonetic boundaries and the degree of auditory-feedback-based speech compensation has previously been demonstrated in a recent study by Niziolek and Guenther (2013), who observed that the magnitude of compensation to real-time formant alterations was larger when perturbations "push" the perceived sound into the region of the boundary between two phonemes (e.g., between $[\varepsilon]$ and $[æ]$ ). While that study did not involve manipulations of lexical status or changes to the phonetic boundary itself (capitalizing, rather, on naturally occurring variation in formant frequencies among repeated productions of the same vowel), it supports the notion that the sensory error signal that drives speech motor compensation is defined, in whole or in part, by the proximity of acoustic output to the phonetic boundary.

The speech adaptation effects obtained in the LC condition are compared to a Non-Lexical-Change (NLC) control condition, in which participants once again produced either $[\varepsilon]$ real words or pseudo-words. However, contrary to the LC condition, the stimuli in this condition were selected such that the F1 shifts did not result in a change in lexical status (i.e., a pseudo-word remained a pseudo-word, and a real-word remained a real-word). We expected that subjects in the NLC condition would not exhibit a difference in speech adaptation depending on whether they produced real words or pseudo-words, owing to the lack of any perceptual boundary shift and/or change in lexical status.

\section{MATERIALS AND METHODS \\ SUBJECTS}

Forty adult subjects were tested (age range: 18-30 years). All were native speakers of English and had no reported history of speech, language or hearing disorder. Hearing status was verified immediately prior to testing using a pure-tone hearing screening (threshold $<20 \mathrm{~dB}$ HL at octave frequencies between 250 and $4000 \mathrm{~Hz}$ ). Subjects provided written informed consent prior to

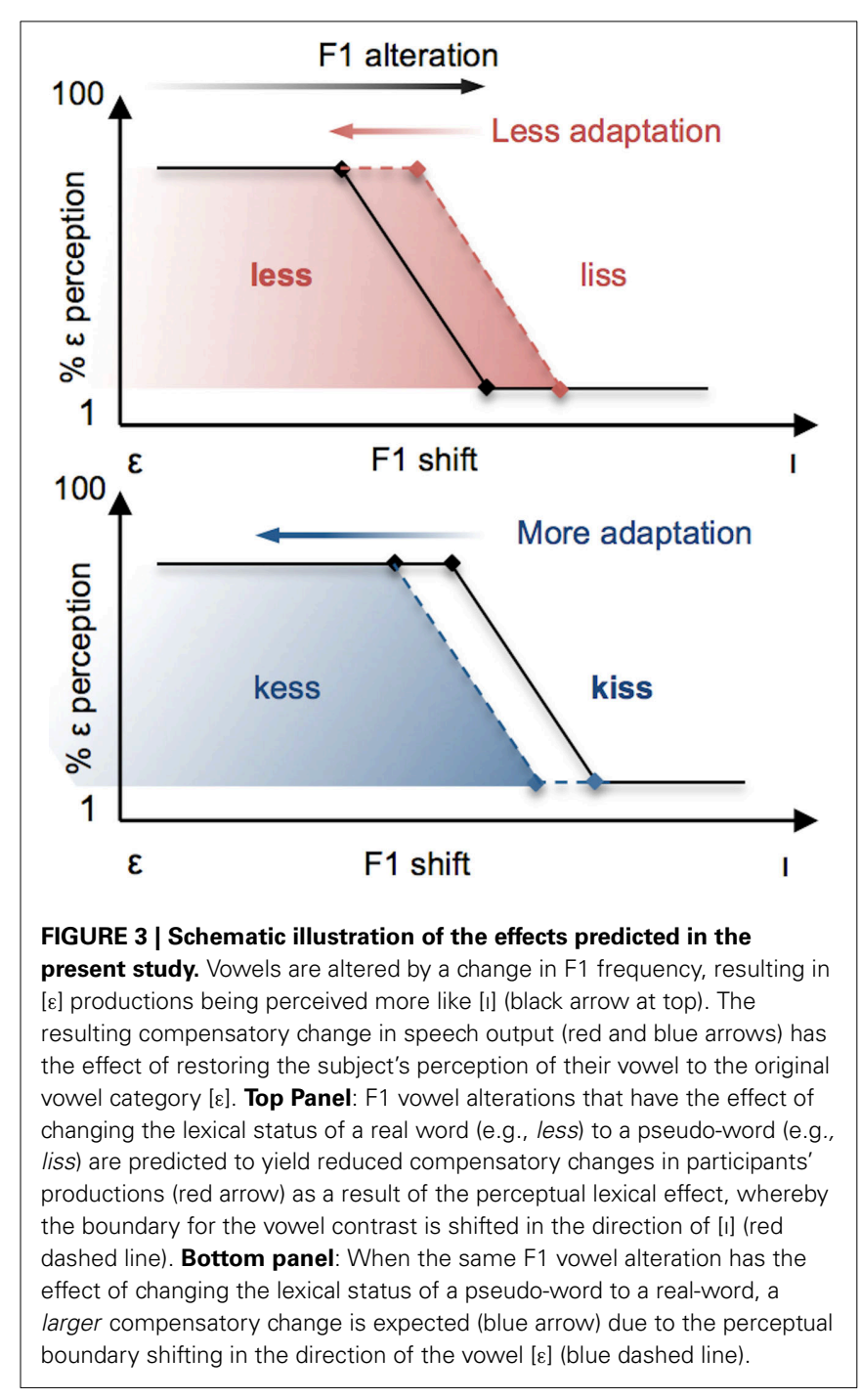

testing. All procedures were approved by the Institutional Review Board, Faculty of Medicine, McGill.

\section{STIMULUS WORDS AND GROUP ASSIGNMENT}

Subjects were randomly assigned to one of four groups (10 subjects in each, 5 males and 5 females), each of which underwent an identical series of speech production tasks (see Table 1 and Procedures below), including the production of monosyllabic words (real-words or pseudo-words) under normal-feedback conditions (baseline task) followed by the production of words under conditions of altered auditory feedback (speech adaptation task). For all four groups, the words produced in the speech adaptation task contained the vowel $[\varepsilon]$ (e.g., "bed" or "geck"). During this task, an acoustic manipulation was carried out in real time such that the vowel $[\varepsilon]$ was perceived to be closer to the vowel [I] (as in "bid"; see Real-time alteration of speech below). The key difference among the four groups relates to the stimuli produced under these conditions: for two of the groups (lexical-change), the stimuli were such that the acoustic manipulation of the vowel changed the lexical status 
of the item: one group ( $L C$-1: word to pseudo-word) produced a set of real-words that, when altered, resulted in a set of pseudo-words (e.g., "test" becoming "tist"), and another group (LC-2: pseudoword to word) produced a set of pseudo-words that, when altered, results in a set of real-words (e.g., "ked" becoming "kid"). For the two other groups (non-lexical change), the stimuli were such that the acoustic manipulation of the vowel did not alter their lexical status: one group (NLC-1: word to word) produced a set of real-words that, when altered acoustically, resulted in another set of real-words (e.g., "bed" becoming "bid"), and a second group (NLC-2: pseudo-word to pseudo-word) produced a set of pseudowords that, when altered acoustically, resulted in a different set of pseudo-words (e.g., "geck" becoming "gick").

For subjects in the two lexical-change groups ( $L C-1$ and $L C-2)$, the stimulus set consisted of 10 pairs of items (see Table 1, left). For subjects in the two non-lexical-change groups (NLC-1 and $N L C$-2), the stimulus set consisted of 9 pairs of items (see Table 1 right). The slight difference in the number of stimuli was due to the difficulty in finding words and pseudo-words that met the required phonetic and lexical criteria for the NLC control groups. For the baseline production task, subjects produced each item 5 times each in a fully randomized order, yielding a total of 100 items for the LC groups, and 90 items for the NLC groups.

The sets of words and pseudo-words used in all of the groups (including the target $[\varepsilon]$-words and the $[\mathrm{I}]$-words resulting from

Table 1 | Distribution of groups as a function of condition.

\begin{tabular}{|c|c|c|c|c|c|c|c|}
\hline \multicolumn{4}{|c|}{ Lexical change } & \multicolumn{4}{|c|}{ No lexical change } \\
\hline \multicolumn{2}{|c|}{ LC1 (G1) } & \multicolumn{2}{|c|}{ LC2 (G2) } & \multicolumn{2}{|c|}{ NLC1 (G3) } & \multicolumn{2}{|c|}{ NLC2 (G4) } \\
\hline$\varepsilon$ & $\mathbf{I}$ & $\varepsilon$ & $\mathbf{I}$ & $\varepsilon$ & I & $\varepsilon$ & I \\
\hline death & dith & weth & with & bet & bit & jex & jix \\
\hline depth & dipth & het & hit & pet & pit & bep & bip \\
\hline nest & nist & fet & fit & ten & tin & tret & trit \\
\hline less & liss & ket & kit & peck & pick & sten & stin \\
\hline chess & chiss & kess & kiss & pen & pin & geck & gick \\
\hline chest & chist & steff & stiff & tech & tick & tetch & titch \\
\hline test & tist & ked & kid & neck & nick & meck & Mick \\
\hline vest & vist & detch & ditch & mess & miss & ness & Niss \\
\hline best & bist & steck & stick & gem & gym & vess & Viss \\
\hline keg & kig & stell & still & & & & \\
\hline
\end{tabular}

the acoustic manipulation) were matched on a number of criteria, including neighborhood density (Pisoni and Tash, 1974), assessing the number of words that are phonologically similar to the target words, and bi-phonemic probability, which represents how frequently the phoneme pairs in the target words occur together in the lexicon (Vitevitch and Luce, 2004; see Table 2). Both of these variables have been shown to influence word production (Munson and Solomon, 2004; Goldrick and Larson, 2008). A Three-Way analyses of variance (ANOVA), including the factors Condition (Lexical change vs. Non-lexical change), Word Type (Word vs. Pseudo-word) and Vowel ([E] vs. [I]) was carried out separately for these two measures. For neighborhood density, all main and interaction effects were not significant $(p>0.05)$. A significant difference in bi-phonemic probability was found between $[\varepsilon]$ and $[\mathrm{I}]$ words $\left[F_{(1,69)}=8.22, p<0.006\right]$ : it was significantly higher in $[\mathrm{I}]$-words relative to $[\varepsilon]$-words $(0.006$ vs. 0.003 ). No significant interactions were obtained (all $p$ 's $>0.06$ ). No reliable difference was observed for this variable between the different lexical change conditions or word-types $(p>0.05)$, however, thus the four groups remained matched.

\section{TESTING PROCEDURES}

Speech was recorded in a quiet testing room using a headmounted microphone (C520, AKG, Germany) and digitized at 16-bit/44.1 kHz on a PC using custom software written in Matlab (Mathworks, MA). Auditory speech signals were presented to subjects using circumaural headphones ( 880 pro, Beyerdynamic, Germany).

All subjects underwent the following sequence of tasks:

1. Baseline speech production: The first task involved the production of a set of stimuli under conditions of normal auditory feedback. Each stimulus was presented orthographically on a computer monitor for $1.5 \mathrm{~s}$, followed by a blank screen for $2 \mathrm{~s}$ between items. Subjects were instructed to produce each item as soon as it appeared on screen. Prior to beginning, subjects practiced producing each stimulus item once and their pronunciation was corrected, if necessary, by the experimenter (for all items, subjects were told to produce the vowel "e" as in the word "head," and the vowel " $\mathrm{i}$ " as in the word "hid").

2. Test of speech motor adaptation: For subjects in each group, the baseline production task was followed by a test of speech

Table 2 | Control measures for experimental stimuli, including neighborhood density (ND) and word-average bi-phonemic probability (PB).

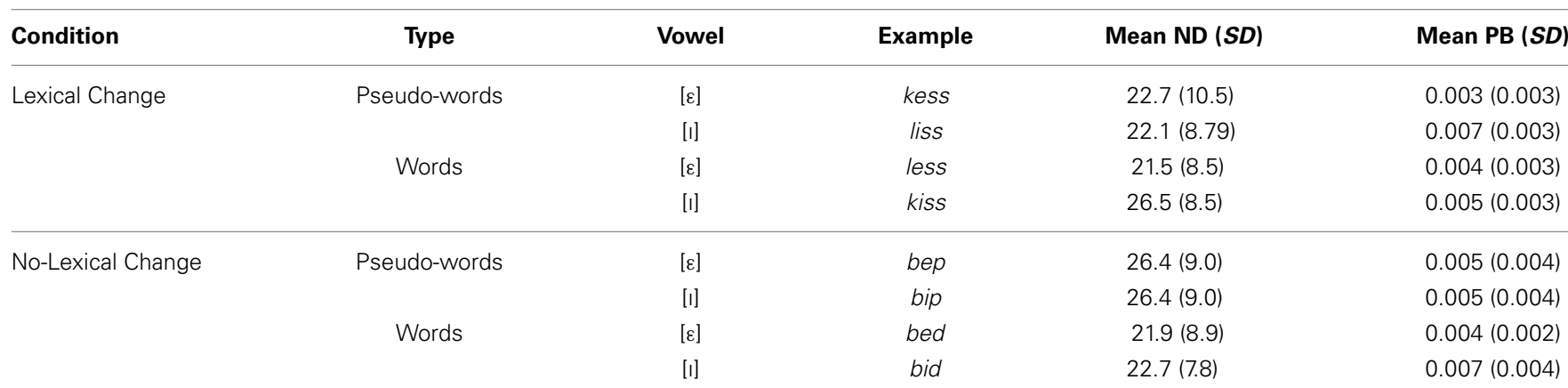


motor adaptation involving 160 productions of words (or pseudo-words) containing the vowel $[\varepsilon]$ from the group's target stimulus list. Word order was randomized. Similar to prior studies of speech adaptation to altered auditory feedback (Purcell and Munhall, 2006; Villacorta et al., 2007; Shiller et al., 2009; Rochet-Capellan and Ostry, 2011), subjects underwent a sequence of auditory feedback conditions involving an initial period of normal feedback (30 trials, null phase), followed by a period of practice under conditions of altered auditory feedback (100 trials, hold phase), and then finally a period under normal feedback once again, to test for the presence of learning after-effects (30 trials, after-effect phase). The auditory feedback manipulation corresponded to a $30 \%$ decrease in the frequency of the first spectral peak (the first formant, or F1), which resulted in a vowel that was perceived to be more like the vowel [I] (see Real-time alteration of speech for details).

\section{REAL-TIME ALTERATION OF SPEECH}

The alteration of auditory feedback involved a 30\% decrease in the first formant (F1) of the vowel acoustic signal (average shift: $216.3 \mathrm{~Hz}$ ). The F1 manipulation was carried out using a system that has been described previously (Rochet-Capellan and Ostry, 2011; Shum et al., 2011; Lametti et al., 2012; Mollaei et al., 2013). The microphone signal was amplified and split into two channels: one providing an unprocessed signal and the other altered using a digital signal processor (DSP) to decrease the frequency of all vowel formants (VoiceOne, TC Helicon). The VoiceOne is a commercial DSP designed to alter human speech signals, using source-filter modeling and re-synthesis to independently manipulate fundamental frequency (F0) and formant frequencies (with all formant frequencies shifted proportionally). While the specific processing algorithm is proprietary, the magnitude of formant manipulations and independence of formant and F0 changes was verified empirically by analyzing input and output signals. The vowel alteration was restricted to $\mathrm{F} 1$ by splitting both signals into non-overlapping low- and high-frequency components (Wavetek 753 low/high pass filter), and then mixing the low-frequency portion of the processed signal with the highfrequency portion of the unprocessed signal. The filter cutoff used to separate the two signals was set at $1100 \mathrm{~Hz}$ for males and $1350 \mathrm{~Hz}$ for females, each of which lies roughly half-way between the first and second formant values for the production of the vowel $[\varepsilon]$ for men and women respectively (based upon pilot studies). The total signal processing delay was less than $15 \mathrm{~ms}$.

Subjects were encouraged to maintain a constant speaking volume throughout the task through the use of a VU meter presented on the computer display (showing current and peak acoustic signal level during each trial). Subjects were instructed to maintain a target level on the display, which was adjusted at the beginning of the experiment to correspond to a comfortable speaking volume. The subject's perception of his or her own air/boneconducted speech acoustic signal was reduced by mixing the auditory feedback signal (presented at approximately $75 \mathrm{~dB}$ SPL) with speech-shaped masking noise (presented at approximately $60 \mathrm{~dB}$ SPL).

\section{ACOUSTIC ANALYSES}

For each word production in the baseline and speech adaptation tasks, a 30 ms segment centered about the midpoint of the vowel was selected using an interactive computer program that displayed the waveform and spectrogram of each utterance, allowing the experimenter to identify a stable, artifact-free region near the vowel center. Mean F1 and F2 (second formant) frequency for each segment was then estimated using LPC analysis in Matlab. LPC parameters were chosen on a per-subject basis in order to minimize the occurrence of clearly spurious formant values. Values of F1 and F2 frequency were used to directly compare vowel acoustic properties during baseline productions of the different stimulus words among the different groups.

Analysis of vowel acoustics during the speech adaptation task was restricted to $\mathrm{F} 1$ frequency, as the compensatory response was primarily observed in this acoustic parameter. During the adaptation task, changes in F2 under conditions of altered auditory feedback rarely exceeded $1 \%$ of baseline values, consistent with other reports of vowel feedback manipulations that were restricted to F1 (see, e.g., Purcell and Munhall, 2006; Villacorta et al., 2007). Following Villacorta et al. (2007) and others (RochetCapellan and Ostry, 2011; Shum et al., 2011; Lametti et al., 2012; Mollaei et al., 2013), changes in vowel production during the speech adaptation task were computed as the proportion change in F1 frequency relative to the mean values during the null phase (averaged over trials 11-30). Such normalized units (which convey changes in formant values relative to a nominal value of 1) are preferable to non-normalized units as they account for individual differences in baseline acoustic properties (e.g., between men and women). Differences in speech adaptation between the different groups were evaluated at three time-points: (1) the beginning of the Hold phase (averaged over trials 31-60) under conditions of altered auditory feedback, (2) the end of the Hold phase (trials 101-130) under conditions of altered auditory feedback, and (3) during the After-Effect phase following removal of the feedback manipulation (trials 131-160).

\section{RESULTS}

\section{BASELINE MEASURES}

Baseline productions of $[\varepsilon]$-words produced in the four different groups were compared in order to verify that mean F1 and F2 values were similar among the different real-word and pseudo-word conditions (thus yielding similar magnitudes of F1 alteration during the speech adaptation task). Mean and SD values of F1 for the two lexical-change groups (LC-1 and LC-2) and the two non-lexical-change control groups (NLC-1 and NLC-2) respectively were: $756.7(97.7), 670.0(132.4), 722.7(105.1)$, and 739.3(122.4) Hz. Mean and SD values of F2 for the four groups respectively were: 1887.6(176.9), 1828.2(162.7), 1831.6(150.9), and $1828.9(195.5) \mathrm{Hz}$. A One-Way ANOVA was carried out to assess any differences among the four conditions in F1 and F2. No significant differences were found between conditions for either formant $\left[\mathrm{F} 1: F_{(3,36)}=1.12, p=0.35 ; \mathrm{F} 2: F_{(3,36)}=0.30\right.$, $p=0.822]$.

\section{SPEECH ADAPTATION}

As shown in Figure 4A, subjects in both lexical-change groups (LC-1, producing real-words, and LC-2, producing 
pseudo-words) exhibited a change in F1 frequency (compensatory increase) in response to the auditory feedback manipulation, though with a notable difference between groups in the magnitude of the response. The compensatory change in F1 can be seen to build up throughout the hold phase and then diminish gradually during the after-effect phase. For the two non-lexicalchange groups (NLC-1, producing real-words, and NLC-2, producing pseudo-words), the time-course of compensatory change in F1 is shown in Figure 5A. For both groups, a robust increase in F1 frequency can be seen following the onset of altered auditory feedback. In contrast with the two LC groups, however, very little difference in the magnitude of compensation is observed throughout the training.

As a first analysis step, we evaluated for each group whether the maximum observed compensatory changes in formant values (at the end of the hold phase) were statistically reliable, using HolmBonferroni-corrected single-sample $t$-tests comparing formant values against a hypothesized mean of 1 (the value representing no difference from baseline in normalized units). Note that in each of the two lexical-change groups (LC-1 and LC-2), a single subject exhibited a change in F1 frequency in the direction opposite that of the compensatory response, as indicated by a statistically reliable F1 decrease at the end of the hold phase (trials $101-130)$ relative to baseline for those two subjects $(p<0.01)$. No subjects in the non-lexical-change groups (NLC-1 and NLC2) exhibited a reliable F1 change in the negative direction. The presence of subjects who show such "following" responses to feedback perturbations has been noted in previous studies (e.g., Villacorta et al., 2007; MacDonald et al., 2010). In the present study, such subjects were excluded from subsequent analyses to avoid averaging across responses that were qualitatively different (negative vs. positive change). All four groups were found to exhibit a reliable compensatory change in $\mathrm{F} 1$ at the end of the hold phase [LC-1: $(8)=2.78, p=0.018$; LC- $2: t_{(8)}=5.30$,
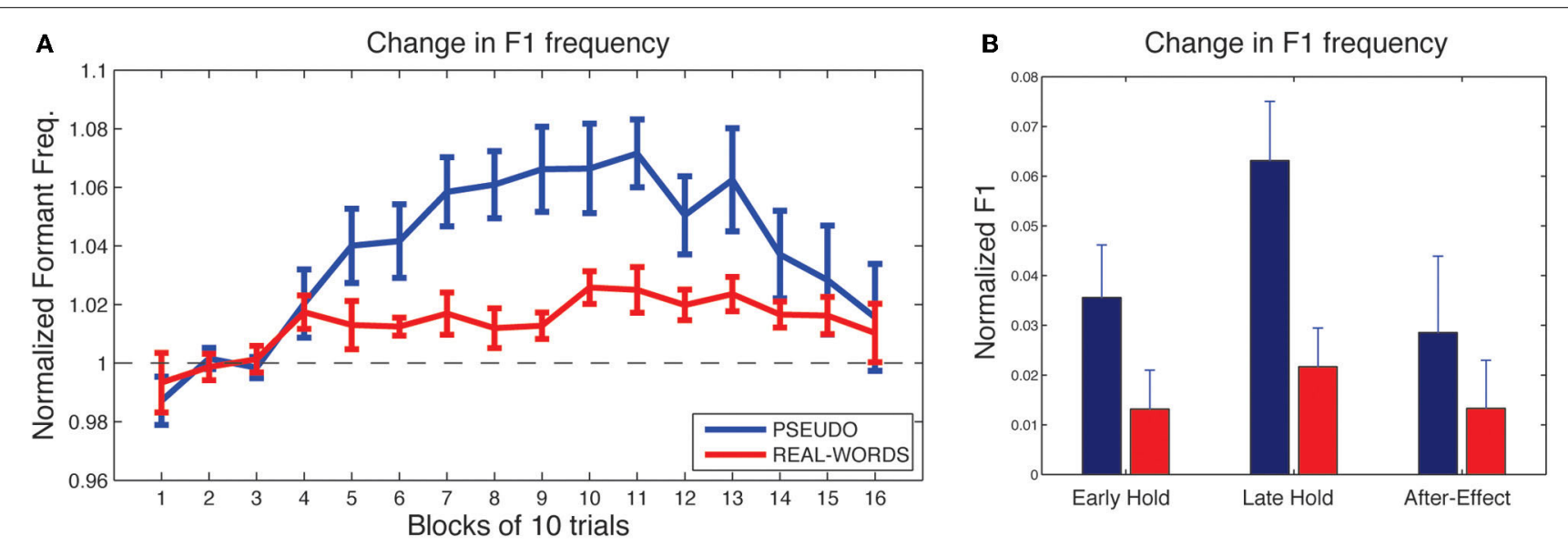

FIGURE 4 | Results of speech adaptation task for the two lexical-change groups (LC-1 and LC-2). (A) Mean F1 frequency (normalized units) is shown throughout the task, averaged over successive blocks of 10 trials each. The magnitude of the change in F1 is notably greater for LC-2 (blue line), compared with LC-1 (red line). (B). Mean normalized F1 at three key time-points during the adaptation task.
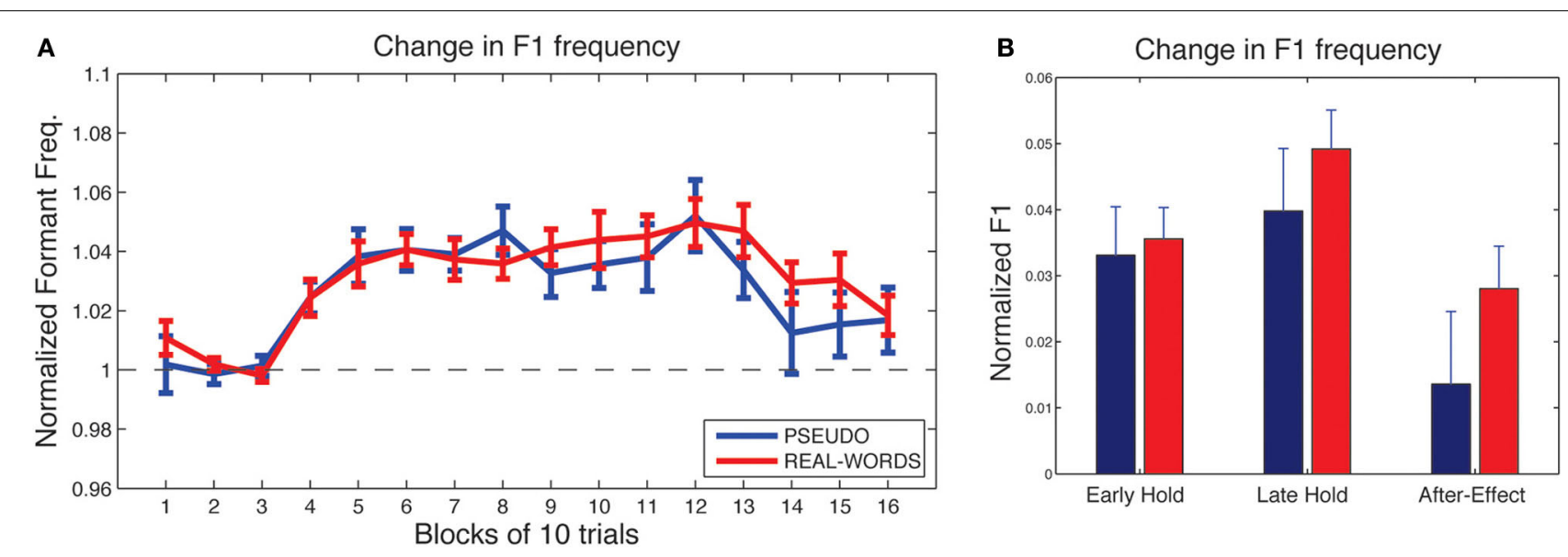

FIGURE 5 | Results of speech adaptation task for the two non-lexical-change groups (NLC-1 and NLC-2). (A) Mean normalized F1 frequency is shown throughout the task, averaged over successive blocks of
10 trials each. The magnitude of the change in $\mathrm{F} 1$ is comparable for the two groups. (B) Mean normalized F1 at three key time-points during the adaptation task. 
$p<0.001 ;$ NLC-1: $t_{(9)}=8.39, p<0.001 ;$ NLC-2: $t_{(9)}=4.2$, $p=0.003]$.

Mean changes in formant values relative to baseline at three time-points during the testing sequence are shown for the lexicalchange groups in Figure 4B, and for the non-lexical-change groups in Figure 5B. To compare the magnitude of the adaptation response among the two lexical-change conditions, the two word-production conditions, and the three time-points, an omnibus Three-Way mixed-factorial ANOVA was carried out with WORD (word vs. non-word) as one between-subjects factor, LEXICAL (lexical change vs. non-lexical-change) as a second between-subjects factor, and PHASE (Early-hold, Late-hold, After-effect) as a within-subjects factor. The two between-subject main effects (WORD and LEXICAL) were found to be nonsignificant [WORD: $F_{(1,34)}=0.87, p=0.36$; PHASE: $F_{(1,34)}=$ $0.22, p=0.64$ ], however a reliable 2-way interaction between WORD and LEXICAL was observed $\left[F_{(1,34)}=4.36, p=0.04\right]$. A highly significant main effect of the within-subject variable PHASE was also observed $\left[F_{(2,68)}=22.49, p<0.001\right]$, but there was no reliable 2-way interaction between PHASE and either of the two group variables [PHASE $\times$ WORD: $F_{(2,68)}=2.52, p=$ 0.09 PHASE $\times$ LEXICAL: $\left.F_{(2,68)}=1.20, p=0.31\right]$. The 3-way interaction was also found to be non-significant $\left[F_{(2,68)}=2.046\right.$, $p=0.14]$.

The 2-way interaction between WORD and LEXICAL conditions is of particular interest, since our main prediction involves a difference in the degree of adaptation between the word and non-word production under the lexical-change condition, with no such difference predicted between word groups under the nonlexical-change condition. Post-hoc pair-wise comparisons were carried out using Holm-Bonferroni-corrected $t$-tests to examine the reliability of these simple group effects. The tests were carried out on adaptation performance at the end of the training period (i.e., the Late-hold phase), as this represented the moment at which speech adaptation was maximal for all groups. A reliable difference in the magnitude of speech adaptation was observed between the groups producing real-words and pseudo-words in the lexical-change condition $\left[t_{(16)}=2.91, p=0.04\right]$, while no significant difference was observed between the two word conditions in the non-lexical-change condition $\left[t_{(18)}=0.84, p=\right.$ $0.41]$. A reliable difference was also observed between the two groups producing real words, one in the lexical-change condition and one in the non-lexical-change condition $\left[t_{(17)}=2.86, p=\right.$ $0.03]$. No such difference was observed between the two groups producing pseudo-words $\left[t_{(17)}=1.55, p=0.28\right]$.

\section{DISCUSSION}

This study investigated the possible interaction between perceptual and lexical information in guiding articulatory movements during spoken speech. Our hypothesis was that compensatory changes in speakers' articulatory patterns following an auditoryfeedback perturbation affecting vowel quality would reflect sensitivity to the lexical status of the words produced. In particular, we predicted that the degree of compensation would vary when the auditory feedback manipulation had an effect on the lexical status of the word being produced (changing a real word into a pseudo-word, or vice versa).
The present findings support this prediction: articulatory compensation to a decrease in F1 inducing a shift from real words to pseudo-words (group LC-1) was found to be significantly less than when the same F1 perturbations provoked a reverse shift from pseudo-words to real words (LC-2). Furthermore, the speech compensatory response for the LC-1 group (producing real-words under conditions of lexical-change) was found to be significantly less than that observed for the group producing realwords under non-lexical-change conditions (NLC-1). Crucially, no such difference in speech compensation was observed between the groups producing real-words and pseudo-words under control conditions in which the same auditory feedback manipulation induced no lexical change (groups NLC-1 and NLC-2 respectively). The observed difference in adaptation magnitude between the two LC groups can be interpreted as an extension of the original lexical effect on phoneme perception demonstrated by Ganong (1980). More precisely, this finding suggests that the lexical effect on perception can also be found in production, resulting from the fact that speakers tend to keep their acoustic speech outcomes within the auditory-perceptual space corresponding to the task-related side of the word/non-word boundary (real words or pseudo-words; see Figure 3).

The conceptual and methodological payoffs of combining altered auditory feedback and lexical bias into a single experimental paradigm are twofold. First, evidence for lexically driven motor adaptation to auditory perturbations demonstrates for the first time a concurrent influence of phonetic and lexical information on the control of spoken speech, indicating that articulatory plasticity is in part constrained by the structure of abstract lexical knowledge. Second, observing a lexical effect through participants' speech productions bypasses the methodological shortcomings associated with explicit perceptual decision-making tasks, and thus strongly supports the view that the lexical influence on perception involves a change in phonetic processing (e.g., Gow et al., 2008), and is not simply the result of a bias in lexically-driven decision-making.

A number of early studies of top-down or contextual influences on phonetic perception indicated that such effects may emerge only in slower reaction time ranges, suggesting that the effect is post-perceptual and thus supporting an independent, input-driven phoneme perception mechanism, free of higherlevel factors (e.g., Fox, 1984; Connine and Clifton, 1987; Cutler et al., 1987; Miller and Dexter, 1988; Burton et al., 1989). While the present study does not allow for a precise characterization of the timing with which lexical knowledge exerts, or stops exerting its influence on speech perceptual or motor functions, the present results are more consistent with the view of a more interactive speech perceptual system, with parallel processing of numerous streams of information, permitting early integration during speech and language processing. This view is also supported by neurobiological evidence for an early interaction between wordform representation areas (e.g., the supramarginal gyrus) and lower-level perceptual cortices (e.g., the superior temporal gyrus) in a time range prior to that of decision-making (Gow et al., 2008).

In current neuro-computational models of the sensorimotor control of speech production, such as the DIVA model 
(Guenther et al., 2006; Tourville and Guenther, 2011) or the State Feedback Control model of speech motor control (Houde and Nagarajan, 2011), changes in auditory feedback, such as those introduced in the present study, result in a mismatch between actual and expected auditory consequences of speech production. This gives rise to an auditory error signal that is used (to varying degrees) to directly alter the control of the oral motor system, as well as to update a predictive feed-forward control mechanism, thus improving subsequent speech motor plans. In the present study, the observed difference in the degree of sensory-motor adaptation between the two LC groups indicates an influence of lexical status on the perception of auditory feedback prior to the establishment of the auditory error signal. Recent MEG studies have shown that auditory feedback processing, including the comparison between actual and predicted acoustic outcomes under conditions of altered auditory feedback, occurs at latencies of less than $100 \mathrm{~ms}$ (Aliu et al., 2009; Niziolek et al., 2013). There is also behavioral evidence of speech compensatory responses to unexpected auditory feedback perturbations at latencies of less than $250 \mathrm{~ms}$ that reflect an influence of the phonetic boundary (Niziolek and Guenther, 2013). Therefore, the present result suggests an influence of lexical status on sensorimotor function at a similarly short latency.

The present findings indicate that the perceptual and motor sub-systems of the speech apparatus interact to a certain extent with higher-order lexical information, although the way in which this interaction takes place and at which stage of the production process remains to be determined. This notion is absent in current neurocognitive models of speech production, such as the DIVA model (Guenther et al., 2006). Hickok (2012) recently proposed a hierarchical psycholinguistic-motor control model of speech, whereby activation of lexical information not only constitutes the starting point toward speech output (in the same vein as earlier models of production, cf. Indefrey and Levelt, 2004), but would also exert a possible influence on the acoustic and somatosensory feedback loops underlying spoken speech. Further work will hopefully yield a more detailed analysis of where and when this interaction takes place. Notice also that lexical-sensorimotor interactions may be present on the somatosensory side of speech production, which would be worth exploring in future studies using real-time somatosensory perturbations during speech (e.g., Tremblay et al., 2008).

\section{ACKNOWLEDGMENTS}

The authors wish to thank Marie-Hélène Tcheuffa-Kamo for her help in data collection and pre-processing. This work was supported by the Natural Sciences and Engineering Research Council of Canada and the National Institutes of Health (NIDCDR01DC012502).

\section{REFERENCES}

Aliu, S. O., Houde, J. F., and Nagarajan, S. S. (2009). Motor-induced suppression of the auditory cortex. J. Cogn. Neurosci. 21, 791-802. doi: 10.1162/jocn.2009.21055

Baars, B. J., Motley, M. T., and McKay, D. G. (1975). Output editing for lexical status in artificially elicited slips of the tongue. J. Verb. Learn. Verb. Behav. 14, 382-391. doi: 10.1016/S0022-5371(75)80017-X
Burton, M. W., Baum, S. R., and Blumstein, S. E. (1989). Lexical effects on the phonetic categorization of speech: the role of acoustic structure. J. Exp. Psychol. Hum. Percept. Perform. 15, 567-575. doi: 10.1037/0096-1523.15.3.567

Burton, M. W., Small, S. L., and Blumstein, S. E. (2000). The role of segmentation in phonological processing: an fMRI investigation. J. Cogn. Neurosci. 12, 679-690. doi: 10.1162/089892900562309

Connine, C. M., and Clifton, C. (1987). Interactive use of lexical information in speech perception. J. Exp. Psychol. Hum. Percept. Perform. 13, 291-299. doi: 10.1037/0096-1523.13.2.291

Costa, A., Roelstraete, B., and Hartsuiker, R. J. (2006). The lexical bias effect in bilingual speech production: evidence for feedback between lexical and sublexical levels across languages. Psychon. Bull. Rev. 13, 972-977 doi: 10.3758/BF03213911

Cutler, A., Mehler, J., Morris, D., and Segui, J. (1987). Phoneme identification and the lexicon. Cogn. Psychol. 19, 141-177. doi: 10.1016/0010-0285(87)90010-7

Fox, R. A. (1984). Effect of lexical status on phonetic categorization. J. Exp. Child Psychol. Hum. Percept. Perform. 10, 526-540. doi: 10.1037/0096-1523.10.4.526

Ganong, W. F. (1980). Phonetic categorization in auditory word perception. J. Exp. Psychol. Hum. Percept. Perform. 6, 110-125. doi: 10.1037/0096-1523.6.1.110

Goldrick, M. and Larson, M. (2008). Phonotactic probability influences speech production. Cognition 107, 1155-1164. doi: 10.1016/j.cognition.2007.11.009

Gow, D. W. Jr., Segawa, J. A., Ahlfors, S. P., and Lin, F.-H. (2008). Lexical influences on speech perception: a Granger causality analysis of MEG and EEG sources estimates. Neuroimage 43, 614-623. doi: 10.1016/j.neuroimage.2008.07.027

Guenther, F. H., Ghosh, S. S., and Tourville, J. A. (2006). Neural modeling and imaging of the cortical interactions underlying syllable production. Brain Lang. 96, 280-301. doi: 10.1016/j.bandl.2005.06.001

Hickok, G. (2012). Computational neuroanatomy of speech production. Nat. Rev. Neurosci. 13, 135-145. doi: 10.1038/nrn3158

Hickok, G., Houde, J. F., and Rong, F. (2011). Sensorimotor integration in speech processing: computational basis and neural organization. Neuron 69, 407-422. doi: 10.1016/j.neuron.2011.01.019

Houde, J. D., and Nagarajan, S. S. (2011). Speech production as state feedback control. Front. Hum. Neurosci. 5:82. doi: 10.3389/fnhum.2011.00082

Houde, J., and Jordan, M. I. (1998). Sensorimotor adaptation in speech production. Science 279, 1213-1216. doi: 10.1126/science.279.5354.1213

Indefrey, P., and Levelt, W. J. M. (2004). The spatial and temporal signatures of word production components. Cognition 92, 101-144. doi: 10.1016/j.cognition.2002.06.001

Lametti, D. R., Nasir, S. M., and Ostry, D. J. (2012). Sensory preference in speech production revealed by simultaneous alteration of auditory and somatosensory feedback. J. Neurosci. 32, 9351-9358. doi: 10.1523/JNEUROSCI.0404-12.2012

Levelt, W. J. M. (1983). Monitoringand self-repair in speech. Cognition 14, 41-104. doi: 10.1016/0010-0277(83)90026-4

Levelt, W. J. M. (1989). Speaking: From Intention to Articulation. Cambridge, MA, MIT Press.

MacDonald, E. N., Goldberg, R., and Munhall, K. G. (2010). Compensations in response to real-time formant perturbations of different magnitudes. J. Acoust. Soc. Am. 127, 1059-1068. doi: 10.1121/1.3278606

Miller, G. A., Heise, G. A., and Lichten, W. (1951). The intelligibility of speech as a function of the context of the test materials. J. Exp. Psychol. 41, 329-335. doi: $10.1037 / \mathrm{h} 0062491$

Miller, J. L., and Dexter, E. R. (1988). Effects of speaking rate and lexical status on phonetic perception. J. Exp. Psychol. Hum. Percept. Perform. 14, 369-378. doi: 10.1037/0096-1523.14.3.369

Mollaei, F., Shiller, D. M., and Gracco, V. L. (2013). Sensorimotor adaptation of speech in Parkinson's disease. Mov. Disord. 28, 1668-1674. doi: $10.1002 / \mathrm{mds} .25588$

Munson, B. and Solomon, N. P. (2004). The effect of phonological neighborhood density on vowel articulation. J. Speech Lang. Hear. Res. 47, 1048-1105 doi: $10.1044 / 1092-4388(2004 / 078)$

Myers, E. B., and Blumstein, S. E. (2008). The neural bases of the lexical effect: an fMRI investigation. Cereb. Cortex 18, 278-288. doi: 10.1093/cercor/bhm053

Niziolek, C. A. and Guenther, F. H. (2013). Vowel category boundaries enhance cortical and behavioral responses to speech feedback alterations. J. Neurosci. 33, 12090-12098. doi: 10.1523/JNEUROSCI.1008-13.2013

Niziolek, C. A., Nagarajan, S. S., and Houde, J. F. (2013). What does motor efference copy represent? Evidence from speech production. J. Neurosci. 33, 16110-16116. doi: 10.1523/JNEUROSCI.2137-13.2013 
Oppenheim, G., and Dell, G. S. (2008). Inner speech slips exhibit lexical bias, but not the phonemic similarity effect. Cognition 106, 528-537. doi: 10.1016/j.cognition.2007.02.006

Pisoni, D. B., and Tash, J. (1974). Reaction times to comparisons within and across phonetic categories. Percept. Psychophys. 15, 285-290. doi: 10.3758/BF03213946

Pitt, M. A. (1995). The locus of the lexical shift in phoneme identification. J. Exp. Psychol. Learn. Mem. Cogn. 21, 1037-1052. doi: 10.1037/0278-7393.21.4.1037

Pitt, M. A., and Samuel, A. G. (1993). An empirical and meta-analytic evaluation of the phoneme identification task. J. Exp. Psychol. Hum. Percept. Perform. 19, 699-725. doi: 10.1037/0096-1523.19.4.699

Purcell, D. W., and Munhall, K. G. (2006). Adaptive control of vowel formant frequency: evidence from real-time formant manipulation. J. Acoust. Soc. Am. 120, 966-977. doi: 10.1121/1.2217714

Rochet-Capellan, A., and Ostry, D. J. (2011). Simultaneous acquisition of multiple auditory-motor transformations in speech. J. Neurosci. 31, 2657-2662. doi: 10.1523/JNEUROSCI.6020-10.2011

Shiller, D. M., Sato, M., Gracco, V. L., and Baum, S. R. (2009). Perceptual recalibration of speech sounds following speech motor learning. J. Acoust. Soc. Am. 125, 1103-1113. doi: 10.1121/1.3058638

Shum, M., Shiller, D. M., Baum, S. R., and Gracco, V. L. (2011). Sensorimotor integration for speech motor learning involves the inferior parietal cortex. Eur. J. Neurosci. 34, 1817-1822. doi: 10.1111/j.1460-9568.2011.07889.x

Tourville, J. A., and Guenther, F. H. (2011). The DIVA model: a neural theory of speech acquisition and production. Lang. Cogn. Process. 26, 952-981. doi: 10.1080/01690960903498424

Tourville, J. A., Reilly, K. J., and Guenther, F. H. (2008). Neural mechanisms underlying auditory feedback control of speech. Neuroimage 39, 1429-1443. doi: 10.1016/j.neuroimage.2007.09.054

Tremblay, S., Houle, G., and Ostry, D. J. (2008). Specificity of speech motor learning. J. Neurosci. 28, 2426-2434. doi: 10.1523/JNEUROSCI.4196-07.2008
Villacorta, V. M., Perkell, J. S., and Guenther, F. H. (2007). Sensorimotor adaptation to feedback perturbations of vowel acoustics and its relation to perception. J. Acoust. Soc. Am. 122, 2306-2319. doi: 10.1121/1.2773966

Vitevitch, M., and Luce, P. A. (2004). A Web-based interface to calculate phonotactic probability for words and nonwords in English. Behav. Res. Methods Instrum. Comput. 36, 481-487. doi: 10.3758/BF03195594

Warren, R. M., and Sherman, G. L. (1974). Phonemic restorations based on subsequent context. Percept. Psychophys. 16, 150-156. doi: 10.3758/ BF03203268

Zion Golumbic, E. M., Poeppel, D., and Schroeder, C. E. (2012). Temporal context in speech processing and attentional stream selection: a behavioral and neural perspective. Brain Lang. 122, 151-161. doi: 10.1016/j.bandl.2011. 12.010

Conflict of Interest Statement: The authors declare that the research was conducted in the absence of any commercial or financial relationships that could be construed as a potential conflict of interest.

Received: 08 January 2014; accepted: 24 March 2014; published online: 17 April 2014. Citation: Bourguignon NJ, Baum SR and Shiller DM (2014) Lexical-perceptual integration influences sensorimotor adaptation in speech. Front. Hum. Neurosci. 8:208. doi: $10.3389 /$ fnhum.2014.00208

This article was submitted to the journal Frontiers in Human Neuroscience.

Copyright (c) 2014 Bourguignon, Baum and Shiller. This is an open-access article distributed under the terms of the Creative Commons Attribution License (CC BY). The use, distribution or reproduction in other forums is permitted, provided the original author(s) or licensor are credited and that the original publication in this journal is cited, in accordance with accepted academic practice. No use, distribution or reproduction is permitted which does not comply with these terms. 